\title{
Two friends with eroded nodules on the ears: atypical fibroxanthoma case report*
}

\author{
Filippo Pesapane ${ }^{1}$ \\ Luisa Lunardon ${ }^{1}$ \\ Raffaele Gianotti ${ }^{1}$
}

\author{
Gianluca Nazzaro ${ }^{1}$ \\ Antonella Coggi ${ }^{1}$
}

DOI: http:/ / dx.doi.org/10.1590/abd1806-4841.20153313

\begin{abstract}
Atypical fibroxanthoma is an uncommon mesenchymal tumor that manifests clinically as a reddish papule or nodule in sun-exposed areas of the body. The clinical presentation is not specific and histology and immunohistochemistry are both necessary for a correct diagnosis. Surgery is the gold standard of therapy. Recurrence and metastasis should be excluded with a follow-up at 6 months, since this tumor should nowadays be considered a medium-grade neoplasm, rather than low-grade as previously believed. We report the case of two friends who came to our hospital during the same period, complaining of very similar lesions. After biopsy and immunohistochemical examination, a diagnosis of atypical fibroxanthoma in both cases was formulated.
\end{abstract}

Keywords: Immunohistochemistry; Sarcoma; Skin neoplasms

\section{INTRODUCTION}

AFX, also termed pseudosarcoma, paradoxical fibrosarcoma or pseudosarcomatous reticulohistiocytoma, is an uncommon superficial fibrohistiocytic tumor. It occurs mainly on sunexposed skin of elderly individuals and shows a male predominance. The lesion usually presents as an asymptomatic, solitary, often dome-shaped nodule, which may be ulcerated. Histologically, AFX consists of an admixture of highly pleomorphic spindle, epithelioid and multinucleated giant cells in the dermis. ${ }^{1}$ The growth pattern of AFX has a propensity of local invasion beyond the involved skin, thereby presenting the risk of local recurrence after surgical excision, which is the gold standard of treatment. ${ }^{2}$

\section{CASE REPORT}

A 78-year-old healthy man was referred to our clinic due to the presence of a juicy-red, dome-shaped nodule. The nodule, $1 \mathrm{~cm}$ in diameter, appeared on

the left antihelix appeared approximately one year before (Figure 1). Physical examination revealed sundamaged skin and a surgical scar due to previous excision of a basal cell carcinoma.

One week later, a friend of his (a 75-year-old man) came to our department with a similar skin lesion. The nodule was oval-shaped, exophytic and bleeding (Figure 2). It was located on the right helix and had a diameter of $1.5 \mathrm{~cm}$. According to the patient, the lesion appeared about 2 months beforehand and had been increasing in size.

In both patients, we performed surgical removal of the lesions, suspecting basal cell carcinomas. Histological examination showed similar findings in both samples such as central erosion associated with pleomorphic spindle and epithelioid cell infiltration in the context of a thickened vascularized stroma (Figure 3 and 4). The proliferating cells were CD68+ (Figure 5), CD163+, FXIII+ and smooth muscle actin+, while melanocytic

Received on 10.12.2013.

Approved by the Advisory Board and accepted for publication on 19.01.2014

Work performed at the Dipartimento di Fisiopatologia medico-chirurgica e dei trapianti, Università degli Studi di Milano - Fondazione IRCCS Ca' Granda Ospedale Maggiore Policlinico - Milano, Italy.

Università degli Studi di Milano - Fondazione IRCCS Ca' Granda Ospedale Maggiore Policlinico - Milano, Italy. 


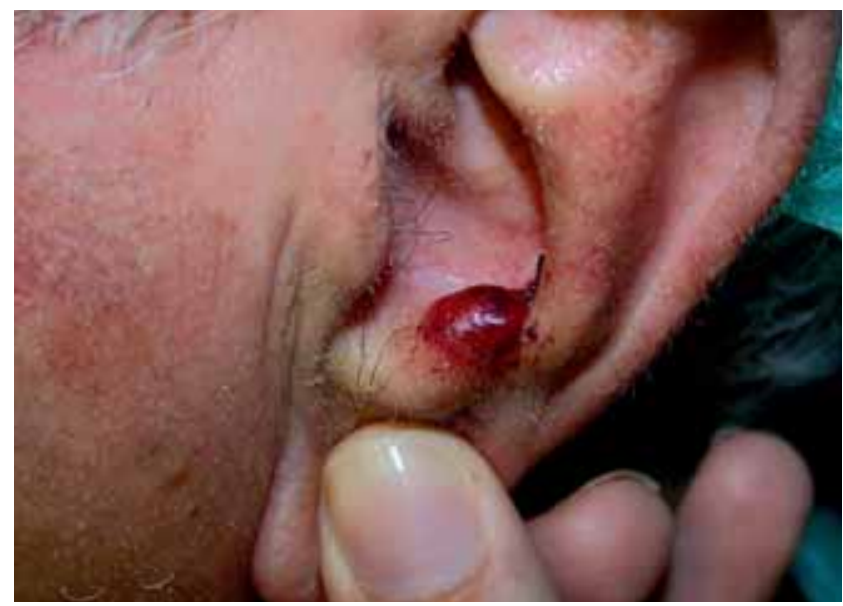

FIGURE 1: A reddish nodule on the left antihelix (first patient)

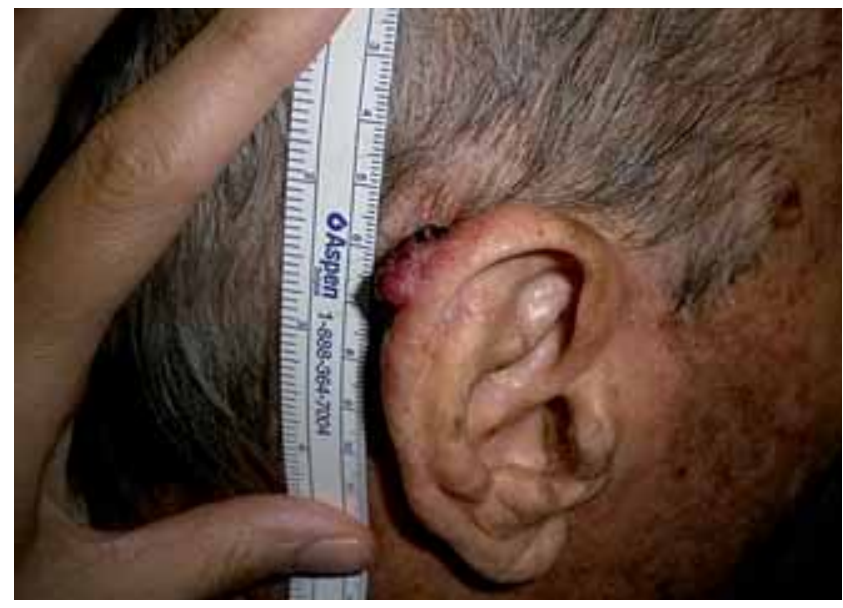

Figure 2: An exophytic nodule on the right helix (second patient)

markers (S100, melan-A, HMB45) were negative and melanoma was therefore excluded. A final diagnosis of atypical fibroxanthoma (AFX) was made in both cases, which was subsequently treated via surgical excision. The 6-month follow-up did not reveal any new lesions in our patients.

\section{DISQUSSION}

AFX is a rare, rapidly growing neoplasm of intermediate malignant potential.

First described by Helwig ${ }^{3}$ in 1961 as a lowgrade dermal tumor with atypical spindle cells, AFX is now generally considered an intermediate malignant potential tumor with a low metastasize-grade ${ }^{2}$. The tumor usually presents as a pink or red papule/nodule, associated or not with erythema and desquamation. Ulceration and bleeding may be present. The tumor is usually skin-colored or brown-red, painless, firm and

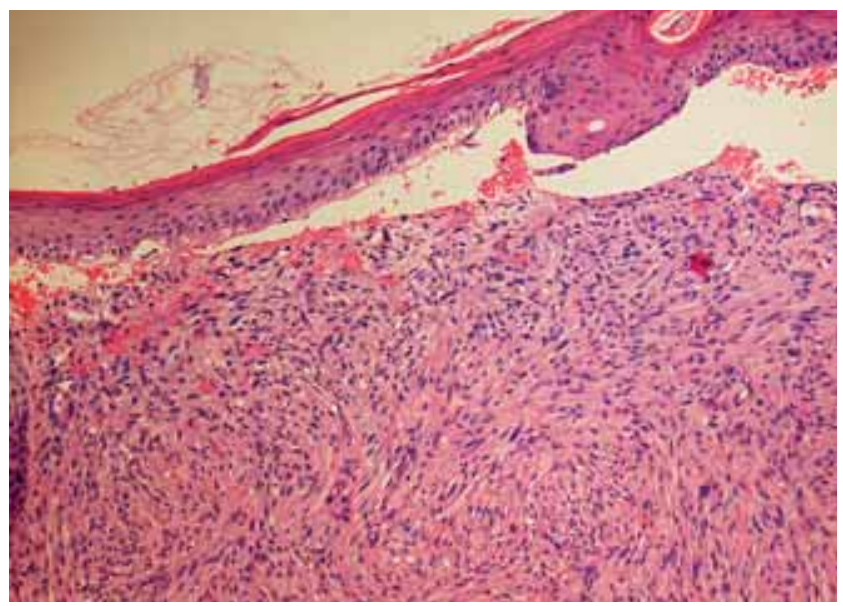

Figure 3: An erosed nodule composed of pleomorphic cells (H.E. $-4 x)$

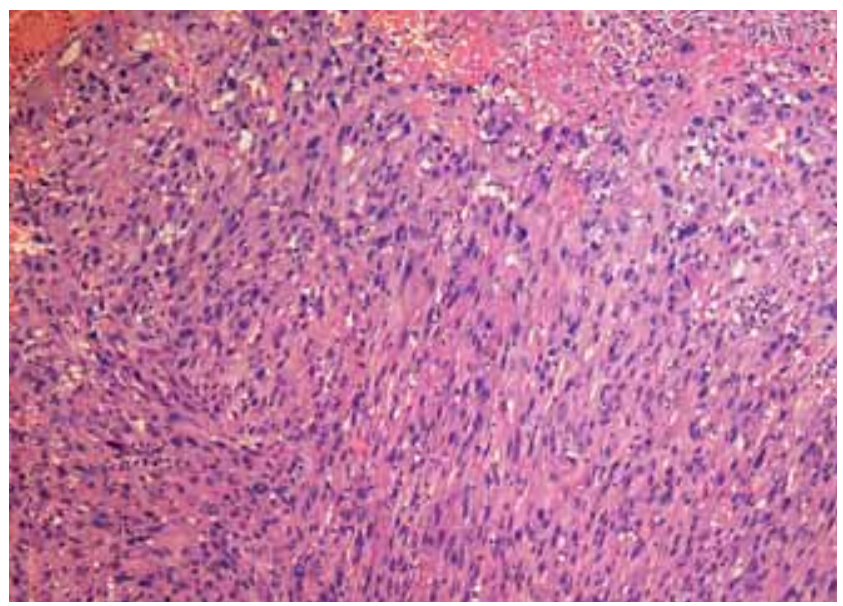

Figure 4: Close-up view of Figure 3 (H.E. - 20x)

frequently ulcerated ${ }^{4}$. Lesions are smaller than $2 \mathrm{~cm}$ in diameter, although the range may vary between $0.3-10 \mathrm{~cm}$. No characteristic dermatoscopic features have been described. Since clinical presentation is not specific, histopathologic examination is essential for diagnosis.

AFX is typically asymptomatic, unilesional and localized in sun-exposed areas (nose, ears and lips) of elderly patients with a higher incidence in the seventh and eighth decade of life ${ }^{4}$.

The pathogenesis is commonly related to ultraviolet exposure. Several studies have assessed a link to p53 protein and demonstrated signature mutations in the p53 gene, occurring from UV radiation $^{5}$. Moreover $\mathrm{x}$-ray exposure also seems to induce AFX development. A study conducted on 140 AFX cases revealed that $4.2 \%$ of lesions had been developing on previous radiation sites ${ }^{1}$. 


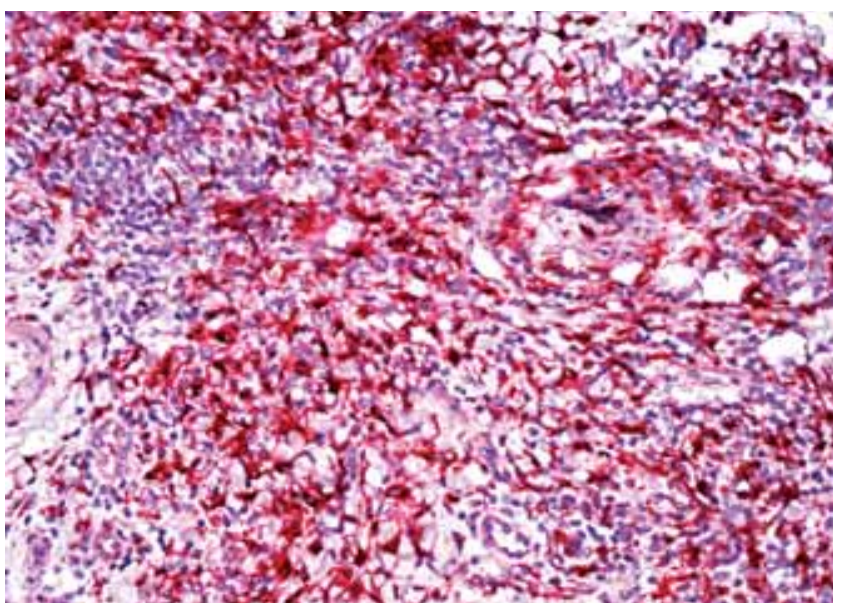

Figure 5: Proliferating cells are CD68-positive CD68 - 20x)

In comparison with typical fibroxanthoma, AFX usually occurs in older individuals (mean age 69) with sun-damaged or radiation-damaged skin on the head, neck or scalp. Men are distinctly more affected, representing between $2 / 3$ and 3/4 of cases ${ }^{6}$. Differential diagnoses include dermatofibrosarcoma protuberans, spindle cell squamous carcinoma and spindle cell malignant melanoma².

Histopathologic examination characteristically reveals a well-circumscribed, exophytic, uncapsulated tumor located in the dermis. No extension to subcutaneous fat is usually observed. The tumor consists of many atypical spindle and epithelioid cells with pleomorphic vesicular or hyperchromatic nuclei. Atypical multinucleated giant cells and mitoses are often seen. Histological characteristics associated with aggressive behavior include involvement of larger portions of subcutis or deep soft tissues, vascular or perineural invasion and the presence of necrosis. The main histological differential diagnoses include spindle cell squamous carcinoma, melanoma and undifferentiated pleomorphic sarcoma. The histological diagnosis of AFX is an exclusion diagnosis. Immunohistochemistry is fundamental to properly diagnose AFX. CD68 and smooth muscle actin are highly positive, while HMB45 and S100 protein are negative ${ }^{4}$.

AFX tumors usually have a benign clinical course. Surgical excision is the therapy of choice. Wide excision with safety margins $(1-2 \mathrm{~cm})$ is recommended as a first choice treatment ${ }^{5}$. In the rare metastatic cases, treatment is patient individualized.

Recurrence and metastasis occur rarely in incompletely excised tumors or when the subcutis or deeper structures are involved and/or tumor necrosis or perineural-vascular invasion is present. Since recurrences usually appear within months to 3 years after primary excision, a clinical follow-up should be performed every 3-6 months over a 2-3-year period. Inspection and palpation of skin, lymph nodes and loco-regional skin, in addition to lymph node sonography, are crucial studies ${ }^{2}$. $\square$

\section{REFERENCES}

1. Fretzin DF, Helwig EB. Atypical fibroxanthoma of the skin. A clinicopathologic study of 140 cases. Cancer. 1973; 31:1541-52.

2. Farley R, Ratner D. Diagnosis and management of atypical fibroxanthoma. Skinmed. 2006; 5:83-6.

3. Helwig EB. Atypical fibroxanthoma: proceedings of the 18th Annual Tumor Seminar of San Antonio Society of Pathologists, 1961. Tex State J Med. 1963; 59:664-7.

4. Beer TW, Drury P, Heenan PJ. Atypical fibroxanthoma: a histological and immunohistochemical review of 171 cases. Am J Dermatopathol. 2010; 32:533-40.

5. Dei Tos AP, Maestro R, Doglioni C, Gasparotto D, Boiocchi M, Laurino L. Ultravioletinduced p53 mutations in atypical fibroxanthoma. Am J Pathol. 1994; 145:11-7.

6 .

Ziemer M. Atypical fibroxanthoma. J Dtsch Dermatol Ges. 2012; 10:537-50.

\author{
M AILING ADDRESS: \\ Filippo Pesapane \\ Via Pace, 9, M ilano. Italy \\ E-mail: filippopesapane@gmail.com
}

How to cite this article: Pesapane F, Nazzaro G, Lunardon L, Coggi A, Gianotti R. Two friends with eroded nodules on the ears: atypical fibroxanthoma case report. An Bras Dermatol. 2015; 90(4):577-9. 